\title{
Parkinson disease risks: correctly identifying environmental factors for a chronic disease
}

\author{
Karl Kieburtz and E. Ray Dorsey \\ Center for Health + Technology and Department of Neurology, University of Rochester Medical Center, Rochester, New York, USA.
}

\begin{abstract}
Parkinson disease (PD) is now the world's fastest growing brain disease; however, the factors underlying this rise are unclear. The past 25 years has witnessed a vast expansion in our understanding of the genetics of $P D$, but few individuals with PD carry one of the major known genetic risk factors. Environmental factors, including individual (e.g., medications) and ambient (e.g., pollutants), may contribute to this rise. In this issue of the $\mathrm{JCl}$, Sasane et al. examined the risk of PD associated with medications commonly used to treat benign prostatic hypertrophy. In contrast with previous studies, certain $\alpha_{1}$ receptor antagonists failed to lower PD risk. Rather, the commonly used comparator drug, tamsulosin, increased PD risk. This finding highlights the importance of selecting comparator groups to correctly identify risk factors. Future studies to address the rise of PD with emphasis on both individual as well as the understudied ambient environmental factors are warranted.
\end{abstract}

\section{A growing brain disease}

Parkinson disease (PD) is the world's fastest growing brain disease, eclipsing the growth of Alzheimer disease (1). From 1990 to 2015 , the number of individuals with PD worldwide has more than doubled, from 2.6 million to 6.3 million (1). Absent change, the number is projected to double again to nearly 13 million in the coming generation (2). Because of its rapid rise, there is increased interest in determining the underlying risk factors for PD. Increasing longevity is a major factor because PD is an age-related disorder that takes decades to unfold. Early features of the disease, such as loss of smell and constipation, can occur ten or more years before classical diagnostic features like tremor develop (3). However, an aging demographic is an insufficient explanation, as the age-adjusted rate of PD is rising (1). The two principal, possibly addressable, PD risks are genetics and environment.

Beginning with the 1997 identification of rare mutations in the $\alpha$-synuclein gene that lead to PD (4), the past 25 years has witnessed an explosion in our understanding of the genetics underlying PD (5). Dozens of genes and genetic risk variants have been identified, but the cumulative result is that the vast majority of individuals (approximately 80\%) with PD do not carry one. By far, the two most common genetic mutations occur in the LRRK2 (leucine-rich-repeat kinase 2) and $G B A$ (glucocerebrosidase) genes (5). However, the former is present in only $3.1 \%$ and the latter in $8.5 \%$ of individuals with PD (6). In addition to these genetic risks, men are at

\section{Related Article: https://doi.org/10.1172/JCI145112}

Conflict of interest: KK has consulted for Clintrex Research Corp, Roche/Genentech, and Novartis, and has ownership interests in Clintrex Research Corp, Hoover Brown LLC, and Blackfynn LLC. ERD has received research support from Biogen, Biosensics, Pfizer, PhotoPharmics, and Wave Life Sciences; compensation for editorial services for Karger Publications; and ownership interests in Grand Rounds (second opinion service).

Copyright: @ 2021, American Society for Clinical Investigation.

Reference information: / Clin Invest. 2021;131(11):e150252. https://doi.org/10.1172/JCI150252.

higher (approximately 40\%) risk for developing the disease, although the explanation remains uncertain. The rapid rise of PD from a rare disease, when Dr. James Parkinson described the shaking palsy in 1817 , to a very common one today, affecting 1.2 million Americans, likely extends beyond a genetic explanation. Recent studies have detailed interactions between genetic factors and environmental causes at the heart of PD (7).

\section{Medications may contribute to $\mathrm{PD}$ risk}

The environment is broad and can be arbitrarily divided into what has been termed "individual" and "ambient" categories (Figure 1). Some individual environmental factors that influence the risk of PD are innate (although modifiable), such as serum urate, with elevated levels conferring a decreased risk of PD (8). Other personal factors are individual behaviors, such as smoking, caffeine consumption, and physical activity, all of which reduce the risk of PD.

Another category of individual environmental risk factor is prescribed medication, a choice shared by individuals and their clinicians. In this issue of the JCI, Sasane et al. explored the impact of the risk of $\alpha_{1}$ adrenergic receptor antagonists on the risk of $\mathrm{PD}$, as previous findings suggest that some drugs in this class may decrease the risk of PD (9). Descriptions of drug classes that may reduce PD risk are not new, with certain $\beta$ adrenergic receptor agonists and nonsteroidal antiinflammatory drugs having been proposed to do the same $(10,11)$. Further analysis of both agents found little or no evidence of a substantive effect on PD risk $(12,13)$. Past mechanistic work and recent epidemiological research (14) suggested that $\alpha_{1}$ adrenergic blockers, which can enhance glycolysis, reduce PD risk compared with tamsulosin, an $\alpha_{1}$ blocker that lacks such a glycolytic effect. While prior work compared just the two groups of $\alpha_{1}$ blockers, 


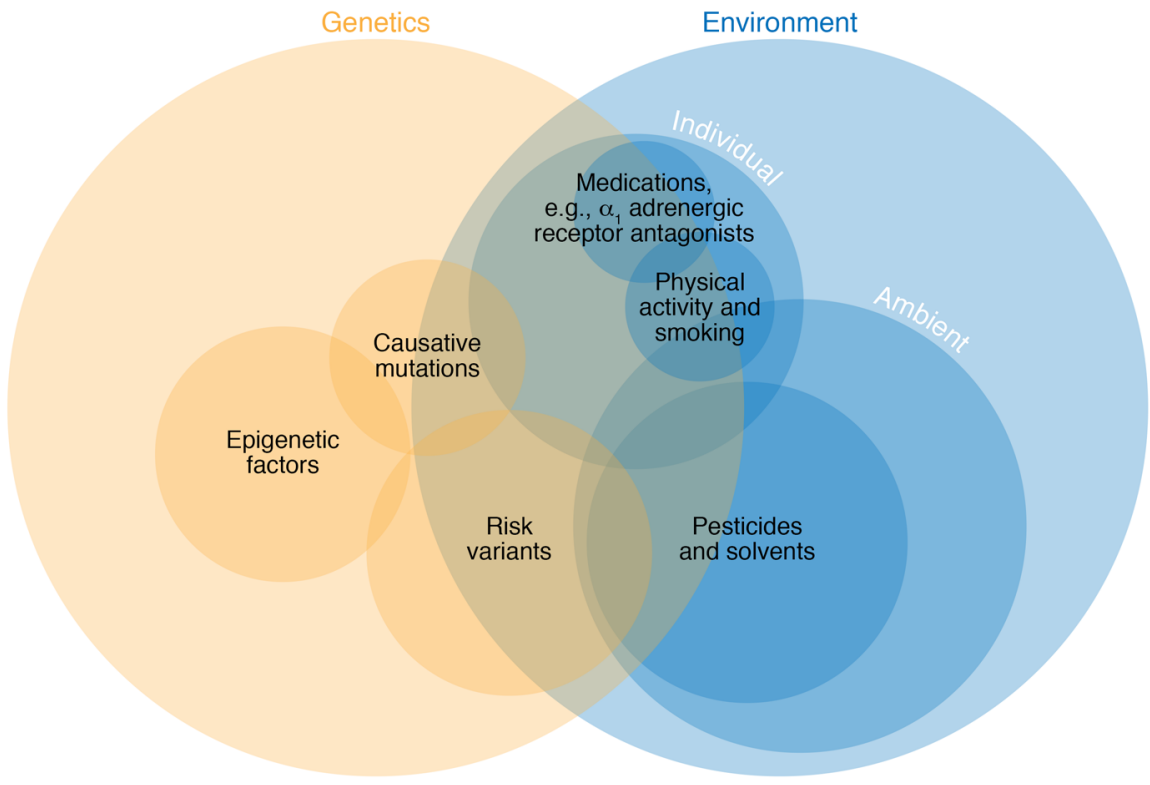

Figure 1. Relationship between genetic and environmental risk factors in Parkinson disease (PD). The interaction between genetic and environmental causes may explain the latency in PD. In this issue of the $J \mathrm{Cl}$, Sasane et al. (9) found that in contrast with previous studies, certain $\alpha_{1}$ receptor antagonists were not protective against PD. Environmental factors, including individual (e.g., medications) and ambient (e.g., pollutants) may contribute to the rising incidence of PD.

Sasane et al. (9) included matched controls not receiving such a drug. Although the two treated groups indeed differed in their PD risk, the risk was elevated in the tamsulosin group, and not reduced with other $\alpha_{1}$ blockers. The earlier findings, suggesting reduced PD risk with $\alpha_{1}$ blockers, have motivated clinical trials of terazosin in parkinsonian disorders looking to slow disease progression (15).

Studies of potential individual environmental risks of PD illustrate the tremendous difficulties in correctly identifying risk factors for a long-latency, late-adultonset chronic disease. Given these complexities, assiduous attention to three details is critical: (a) defining the outcome of interest (e.g., PD), (b) defining the risk of interest (e.g., medication use) and accounting for other known risks (e.g., smoking), and (c) establishing a comparator group (e.g., unexposed to a medication). Sasane et al. (9) included a key comparator group, a matched control cohort without $\alpha_{1}$ blocker exposure, which established a reference comparison point for the two groups exposed to the different types of $\alpha_{1}$ blockers. This additional comparison provided a crucial insight, at least questioning, and likely reversing, prior understanding of $\alpha_{1}$-receptor blocker risks in PD. That said, even if tamsulosin increases the risk of the disease (too early to conclude), its effect is confined to men, largely later in life, and is not a major explanation of the rise of PD.

\section{Ambient environmental causes}

If iatrogenic environmental factors are not fueling the rise of PD, then ambient ones may be. Some of these factors have been identified. Numerous epidemiological and animal studies have implicated certain pesticides, many of which damage mitochondria that show dysfunction in PD. For example, epidemiological studies indicate that exposure to the pesticide paraquat increases the risk of PD by $150 \%$ (16), and the pesticide also reproduces features of the disease when given to mice. Another chemical, the industrial solvent trichloroethylene (TCE), was ubiquitous in the 1970s when it was used in everything from decaffeinating coffee to degreasing automobile parts to providing anesthesia to pregnant women, has also been linked to PD. A study involving twins found that those who had occupational exposure to TCE had $500 \%$ increased risk of developing PD compared with their unexposed twin (17). Moreover, TCE when fed to laboratory animals also reproduced key features of the disease.
Despite these powerful indications of ambient environmental causes for PD, they, as a whole, have been under-investigated. Studies of the environmental risks underlying PD lag genetic studies by a factor of seven or more. The limited investigation of TCE is especially concerning. TCE contaminates up to $30 \%$ of groundwater in the United States, is present in half of the most toxic contamination sites (known as Superfund sites) in the country, pollutes thousands of other sites, and is increasingly used around the world (18). Additional epidemiologic studies of PD will help shed light on the environmental etiologies (individual and ambient) of the disease and on potential means of prevention.

The Sasane et al. (9) study provides a cautionary tale regarding premature conclusions about the link between exposure to medications and the risk of PD. Clinical trials seeking to use medications associated with a decreased risk of PD as treatments for manifest illness have been disappointing to date (e.g., with inosine to raise serum urate; ref. 19). The Sasane et al. (9) study also offers researchers the chance to step back and consider the factors, especially ambient environmental ones, that may drive this, to date, largely silent pandemic (2).

\section{Acknowledgments}

We thank Meghan Pawlik for her assistance in preparing this manuscript.

Address correspondence to: Karl Kieburtz, 265 Crittenden Boulevard, CU 420694, Rochester, New York 14642, USA. Phone: 585.275.8911; Email: karl.kieburtz@chet. rochester.edu.

1. Dorsey ER, et al. Global, regional, and national burden of Parkinson's disease, 1990-2016: a systematic analysis for the Global Burden of Disease Study 2016. Lancet Neurol. 2018;17(11):939-953.

2. Dorsey ER, Bloem BR. The Parkinson pandemic-a call to action. JAMA Neurol. 2018;75(1):9-10.

3. Savica R, et al. When do $\alpha$-synucleinopathies start? An epidemiological timeline: a review. JAMA Neurol. 2018;75(4):503-509.

4. Polymeropoulos $\mathrm{MH}$, et al. Mutation in the alpha-synuclein gene identified in families with Parkinson's disease. Science. 1997;276(5321):2045-2047.

5. Hernandez DG, et al. Genetics in Parkinson disease: mendelian versus non-Mendelian inheritance. J Neurochem. 2016;139(suppl 1):59-74.

6. Skrahina V, et al. The Rostock International Par- 
kinson's Disease (ROPAD) study: protocol and initial findings. Mov Disord. 2021;36(4):1005-1010.

7. Lee J-W, Cannon JR. LRRK2 mutations and neurotoxicant susceptibility. Exp Biol Med (Maywood). 2015;240(6):752-759.

8. Weisskopf M, et al. Plasma urate and risk of Parkinson's disease. Am J Epidemiol. 2007;166(5):561-567.

9. Sasane, et al. Parkinson disease among patients treated for benign prostatic hyperplasia with $\alpha_{1}$ adrenergic receptor antagonists. JClin Invest. 2021;131(11):e145112.

10. Gronich N, et al. $\beta 2$-adrenoceptor agonists and antagonists and risk of Parkinson's disease. Mov Disord. 2018;33(9):1465-1471.

11. Gao X, et al. Use of ibuprofen and risk of Parkin- son disease. Neurology. 2011;76(10):863-869.

12. Kia DA, et al. Mendelian randomization study shows no causal relationship between circulating urate levels and Parkinson's disease. Ann Neurol. 2018;84(2):191-199.

13. Hopfner F, et al. $\beta$-Adrenoreceptors and the risk of Parkinson's disease. Lancet Neurol. 2020;19(3):247-254.

14. Simmering JE, et al. Association of glycolysisenhancing $\alpha-1$ blockers with risk of developing Parkinson disease. JAMA Neurol. 2021;78(4):407-413.

15. Schultz J. Terazosin for Parkinson's Disease (TZ-PD). https://clinicaltrials.gov/ct2/show/ NCT03905811?term=terazosin \& cond $=$ Parkinson+Disease \&draw=2\&rank=1. Updated February 9, 2021. Accessed May 6, 2021.
16. Tanner CM, et al. Rotenone, paraquat, and Parkinson's disease. Environ Health Perspect. 2011;119(6):866-872.

17. Goldman SM, et al. Solvent exposures and Parkinson disease risk in twins. Ann Neurol. 2012;71(6):776-784.

18. De Miranda BR, et al. The industrial solvent trichloroethylene induces LRRK2 kinase activity and dopaminergic neurodegeneration in a rat model of Parkinson's disease. Neurobio Dis. 2021;153:105312.

19. Schwarzschild MA. Study of Urate Elevation in Parkinson's Disease, Phase 3 (SURE-PD3). https://clinicaltrials.gov/ct2/show/results/ NCT02642393. Updated July 28, 2020. Accessed May 6, 2021. 